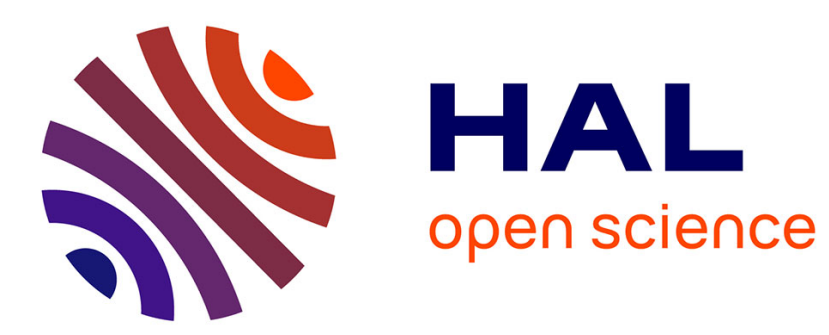

\title{
Special issue on Intelligent Vision Systems
}

Monique Thonnat

\section{To cite this version:}

Monique Thonnat. Special issue on Intelligent Vision Systems. Computer Vision and Image Understanding, 2010, 114 (5), pp 501-608. inria-00502843

\section{HAL Id: inria-00502843 https://hal.inria.fr/inria-00502843}

Submitted on 15 Jul 2010

HAL is a multi-disciplinary open access archive for the deposit and dissemination of scientific research documents, whether they are published or not. The documents may come from teaching and research institutions in France or abroad, or from public or private research centers.
L'archive ouverte pluridisciplinaire HAL, est destinée au dépôt et à la diffusion de documents scientifiques de niveau recherche, publiés ou non, émanant des établissements d'enseignement et de recherche français ou étrangers, des laboratoires publics ou privés. 


\section{CVIU special issue: Intelligent Vision Systems: editorial}

The explosive growth in the number of video cameras and the increasing computing power of computers extend the potential number of applications of vision algorithms. It brings also new challenges to the vision community. In particular the design of intelligent and robust vision systems becomes a rising topic for both researchers and developers from academic fields and industries worldwide. With this special issue we intend to cover all aspects of Intelligent Vision Systems. By this we mean vision systems that interact with or respond to their environment in a dynamic and adaptive manner, with an emphasis put on integrated systems that are robust enough to be deployed in largely unconstrained environments.

This special issue was motivated by the proceedings of the 5th International Conference on Computer Vision Systems (ICVS'07) held in 2007 at Bielefeld University, Germany on March 21st23rd. The major topics were cognitive vision techniques for scene analysis, semantic interpretation and learning as well as computer vision from a system perspective including architecture, integration, control and applications. Thirty one papers were submitted for review and possible inclusion in the special issue. Each of the submitted manuscripts was reviewed by experts in the field of intelligent vision systems. In January 2009, the first rigorous review round was completed, and 20 papers were removed from consideration for the special issue. Papers selected for the inclusion in the special issue underwent two additional review rounds before their definite acceptance. This special issue on intelligent vision systems is composed of eight papers, among which six are extended versions of ICVS07 papers, one an extended version of an ICCV07 paper and an ICVS07 paper and one an extended version of a CVPR06 paper and an IJCAI07 paper.

In this issue five papers cover the various aspects of cognitive vision techniques. The first two papers present high level activity recognition techniques for human interaction. The two following papers show how bio-inspired techniques enable to build practical vision systems. The fifth paper combines unsupervised and supervised machine learning techniques for object categorization. The remaining three papers are concerned by the building of integrated systems and issues related to their robustness towards unconstrained environment.

The first paper by J. Hoey et al is entitled "Automated Handwashing Assistance For Persons With Dementia Using Video And A Partially Observable Markov Decision Process" It received the best paper award of ICVS 07. This paper presents a real-time vision-based system to assist a person with dementia wash his/her hands. The system combines a Bayesian sequential estimation framework for tracking hands and towel, with a decision theoretic framework for computing policies of action.

The paper by M.Ryoo et al is entitled "A Task-Driven Intelligent Workspace System to Provide Guidance Feedback". In this paper they present a vision-based human-computer interaction system which collaborates with a user by providing feedback during user activities. An intelligent workspace system analyzes the context and generates appropriate feedback guiding the user to complete the tasks 
correctly.

The paper by K Shubina and J.Tsotsos is entitled "Visual Search for an Object in a 3D Environment using a Mobile Robot" It describes a particular example of a practical robotic vision system. Visual attention processes are used for finding an object in a mostly unknown space with a mobile robot.

The paper by $\mathrm{T}$ Michalke et al is entitled "A Biologically-Inspired Vision Architecture for Resource-Constrained Intelligent Vehicles" Using a tunable visual attention system and state-of-the-art perception algorithms, the system is capable of analyzing the scenery for task-relevant information in order to provide the driver with assistance in dangerous situations.

The paper by M Fritz et al is entitled "Tutor-Based Learning of Visual Categories using Different Levels of Supervision" They propose a novel method that uses unsupervised training to obtain visual groupings of objects and a cross-modal learning scheme to overcome inherent limitations of purely unsupervised training.

The paper by Seon Joo Kim et al is entitled "Joint Radiometric Calibration and Feature Tracking System with an Application to Stereo" They model the global and nonlinear process that is responsible for the changes in image brightness rather than adapting to the changes locally and linearly which makes the tracking more robust to the change in brightness.

The paper by D Geronimo et al is entitled "2D-3D Based On-Board Pedestrian Detection System" In this paper they present a three module system based on both 2D and 3D cues. The major difficulties are processing outdoor scenes from a mobile platform and searching for aspect-changing objects in cluttered environments.

The paper by H Ekenel is entitled "A Video-based Door Monitoring System Using Local Appearance-based Face Models" In this application many challenges need to be handled for successful recognition:

continuous, uncontrolled variations of facial appearance due to illumination, pose, expression, and occlusion of non cooperative subjects. Extensive experiments have been conducted on the data collected under real-life conditions.

We believe that this special issue is timely and provides a great opportunity for bringing together experts of computer vision, cognitive vision and vision systems. We hope it may serve as a starting point for fruitful debates on the various aspects of intelligent vision systems.

We thank journal manager Linda Shapiro for her help throughout the editorial process, and Avi Kak for his encouragement and support of the project. We thank all the reviewers for their efforts and time and more specially Prof Gerhard Sagerer the general chair of ICVS 2007.

Guest Editor

Monique Thonnat

Deputy scientific director

INRIA Sophia Antipolis

France 